\title{
Infiltración electroforética de nanopartículas de plata en depósitos nanoestructurados de $\mathrm{TiO}_{2}$
}

\author{
Armando Rodríguez ${ }^{\mathrm{a}}$, Gabriela Amor ${ }^{\mathrm{a}}$, Manuel Ceballos ${ }^{\mathrm{a}}$, Israel López ${ }^{\mathrm{a}}$, Alejandro Vázquez ${ }^{\mathrm{a} *}$ \\ ${ }^{a}$ Laboratorio de Materiales I, Facultad de Ciencias Quimicas, Universidad Autónoma de Nuevo León, San Nicolás de los Garza, N.L., \\ México \\ *E-mail: alejandro.lqi@gmail.com
}

Recibido 1 de diciembre de 2014, Aceptado 12 de diciembre 2014

\section{Resumen}

En el presente trabajo se muestra la infiltración electroforética de nanopartículas de plata sobre depósitos de $\mathrm{TiO}_{2}$ los cuales fueron caracterizados por microscopía electrónica de barrido, espectrometría UV-Vis, dispersión dinámica de luz en el cual se observó que los depósitos de $\mathrm{TiO}_{2}$ que se obtuvieron tenían un espesor entre 7 y $11 \mu \mathrm{m}$, también que a mayor tiempo de deposición se formaban aglomerados de plata más grandes, se observó una infiltración en los depósitos aproximada de 3 $\mu \mathrm{m}$, además en todos los tiempos se lograron observar partículas de plata en el sustrato de aluminio debido a que se infiltra por las grietas formadas en el depósito de $\mathrm{TiO}_{2}$.

Palabras clave: deposición electroforética, nanopartículas, plata, dióxido de titanio.

\begin{abstract}
In this work is presented the electrophoretic infiltration of silver nanoparticles in $\mathrm{TiO}_{2}$ films, which were characterized by means of scanning electron microscopy, UV-Vis spectrophotometry, and dynamic light scattering. The $\mathrm{TiO}_{2}$ films have a thickness between 7 and $11 \mu \mathrm{m}$. It was observed that to longer times of deposition the silver agglomerate sizes were higher, it was observe infiltration around $3 \mu \mathrm{m}$ in the coatings; besides, in all the deposition times it was observed silver nanoparticles onto the aluminum substrate, which is due to the infiltration in the cracks formed by $\mathrm{the}^{\mathrm{TiO}} 2$ deposition.
\end{abstract}

Keywords: electrophoretic deposition, nanoparticles, silver, titanium dioxide.

\section{Introducción}

En la técnica de DEF se tiene una suspensión de partículas cargadas, a las cuales se les hará incidir una diferencia de potencial conocida, lo que provoca que las partículas migren al electrodo de carga contraria y comiencen a formar aglomerados de estas, eso es lo que se llama depósito ${ }^{[1]}$; esta técnica tiene la facilidad de ser escalable a nivel industrial debido a que es una técnica sencilla, el equipo es accesible y requiere bajos $\operatorname{costos}^{[2]}$.

El $\mathrm{TiO}_{2}$ es un semiconductor que se utiliza ampliamente en la medicina, microelectrónica, la industria de pintura, papel, etc. Dentro de sus diversas aplicaciones se hará referencia a una de las más comunes que es como fotocatalizador. Un fotocatalizador es aquel material semiconductor que al ser irradiado con luz hará que un electrón de la banda de valencia salte a la banda de conducción eso es lo que se conoce como par electrónhueco (par e-h) que es esos 2 lugares donde se dará la oxidación o reducción para la formación de los radicales $\mathrm{OH}^{*}$ que son los necesarios para la degradación del contaminante (colorantes, detergentes, etc. $)^{[3]}$.
Se han realizado diversos estudios para mejorar la capacidad catalítica del $\mathrm{TiO}_{2}$, y se ha reportado en diversos artículos que con algunos metales como paladio, platino, oro y plata se puede aumentar esa capacidad. Las nanopartículas de dichos metales atrapan los electrones de los pares e-h haciendo ese proceso más duradero, lo que hará que el $\mathrm{TiO}_{2}$ tenga un aumento en su poder catalítico $^{[4]}$. Este trabajo presenta la infiltración de plata con ayuda de la DEF a diferentes tiempos, para su uso potencial como fotocatalizador en la degradación de colorantes.

\section{Experimental}

\subsection{Materiales}

Los reactivos utilizados durante todo el trabajo fueron de grado analítico y sin ninguna purificación previa. Un espectrofotómetro Perkin-Elmer lambda 12 fue utilizado para los análisis UV-Vis. Los depósitos fueron analizados por el microscopio electrónico de barrido (SEM) JEOL JSM-6510LV. Dispersión dinámica de luz (DLS) Zetatrac Microtrac para medir el tamaño de partículas. 
Se preparó una suspensión de $\mathrm{TiO}_{2}$ al $1 \% \mathrm{P} / \mathrm{V}$ colocando $1 \mathrm{~g}$ de $\mathrm{TiO}_{2}$ en $100 \mathrm{~mL}$ de agua con $0.5 \mathrm{~mL}$ de $\mathrm{HNO}_{3}$, esta suspensión se colocó en baño de ultrasonido por $10 \mathrm{~min}$, se dejó reposar por $10 \mathrm{~min}$ y se volvió a colocar en ultrasonido por otros $10 \mathrm{~min}$. Se tomaron $5 \mathrm{~mL}$ de esta suspensión y se añadieron a $45 \mathrm{~mL}$ de agua para formar una suspensión $0.1 \%$ de $\mathrm{TiO}_{2}$, la cual, de la misma forma, se colocó en ultrasonido por $10 \mathrm{~min}, 10 \mathrm{~min}$ de descanso y $10 \mathrm{~min}$ de ultrasonido.

Se tomaron $7 \mathrm{~mL}$ los cuales se procedieron a depositar en el equipo (Fig. 1) durante 24h en placas de aluminio a una distancia de $1 \mathrm{~cm}$ previamente pulidas a $600 \mathrm{mV}$

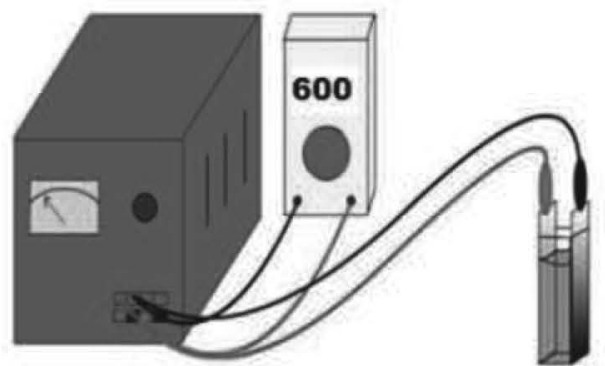

Figura 1. Representación esquemática del equipo de DEF

\subsection{Preparación de depósitos $\mathrm{TiO}_{2} / \mathrm{Ag}$}

Se prepararon soluciones 2milimolar de los siguientes reactivos $\mathrm{AgNO}_{3}, \mathrm{NaBH}_{4}$, Citrato de sodio; se añadieron $2 \mathrm{~mL}$ de citrato y de $\mathrm{AgNO}_{3}$ a $44 \mathrm{~mL}$ de agua destilada y se llevó a agitación magnética, mientras se tenía en agitación se agregaron $2 \mathrm{~mL}$ de $\mathrm{NaBH}_{4}$ lentamente gota a gota; una vez agregado todo el $\mathrm{NaBH}_{4}$ se mantuvo la agitación por 2 min y después se dejó en reposo, mientras no se utilizaba la solución se guardaba en frascos oscuros para evitar la fotoconversión de la plata.

La solución se colocó en el equipo de DEF, se utilizaron los electrodos con depósito de $\mathrm{TIO}_{2}$, se cambió el orden de los electrodos ya que la plata se deposita en el de carga opuesta al $\mathrm{TiO}_{2}$, a diferentes tiempos $(6,12$, 18 horas) a $600 \mathrm{mV}$.

\section{Resultados y discusión}

\subsection{Caracterización de las nanopartículas de $\mathrm{TiO}_{2}$}

En la figura 2 se muestra la imagen obtenida por SEM del depósito de $\mathrm{TiO}_{2}$. En dicha imagen se puede observar la morfología del depósito, además, se aprecian unas grietas que es donde posiblemente se infiltrará la plata con mayo facilidad.

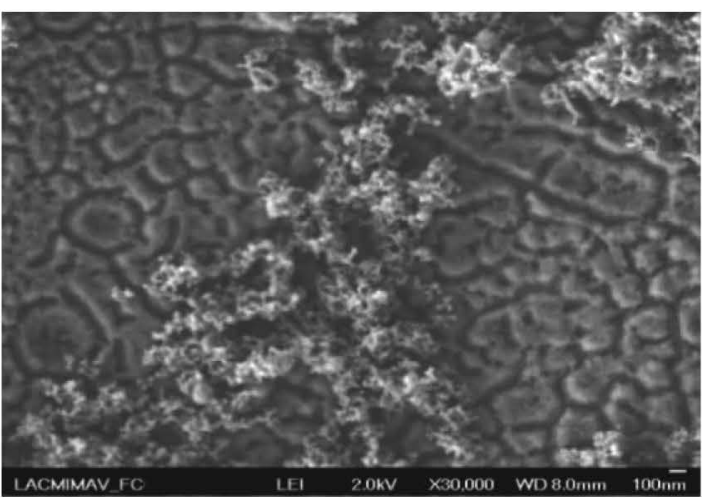

Figura 2. Imagen de SEM de las nanopartículas de $\mathrm{TiO}_{2}$.

\subsection{Caracterización de las nanopartículas de Ag.}

En la figura 3 se muestra un espectro UV-Vis de las nanopartículas de plata, en el cual se puede apreciar que a los $400 \mathrm{~nm}$ se presenta la banda de absorción de la resonancia del plasmón superficial de la plata; además se realizó DLS para corroborar el tamaño de partícula que resultó en promedio de $25 \mathrm{~nm}$.

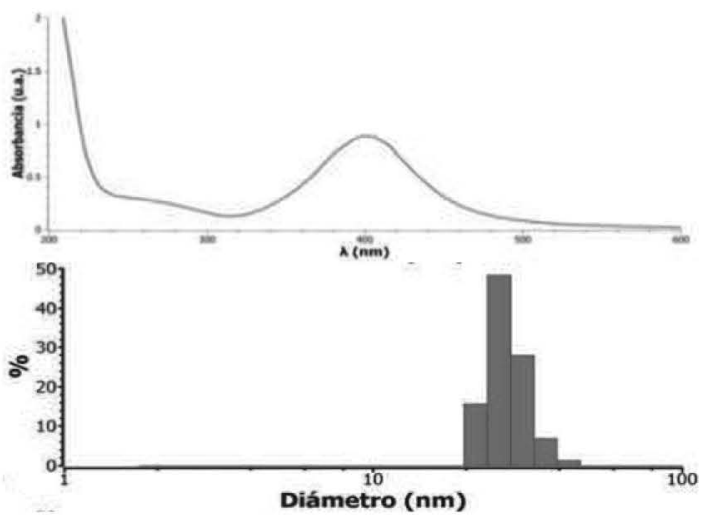

Figura 3. Espectro UV-Vis y DLS de nanopartículas de plata.

\subsection{Caracterización de los depósitos de TiO2/Ag.}

En la figura 4 se muestran las imágenes de SEM de las morfologías obtenidas de los depósitos de plata a 12 y 18 horas, en el cual se observan aglomerados de mayor tamaño en la de 18h; además se puede observar que la mayoría de las grietas en los depósitos desaparecieron debido a que las nanopartículas de plata sí se están depositando y se puede decir que se están infiltrando.

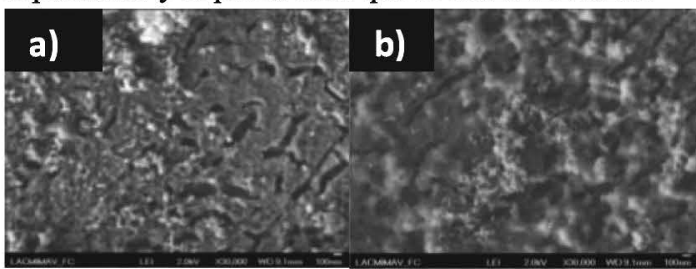

Figura 4. Imagen de SEM de a) 12 horas y b) 18 horas depositando plata.

En la figura 5 se muestra la misma imagen tomada en SEM con diferente detector; detector de electrones 
secundarios (ES) y detector de electrones retrodispersados (ER) y se puede observar el aglomerado un poco más brilloso en el de ER debido a que depende del número atómico lo que nos indica que ese apartado es plata.

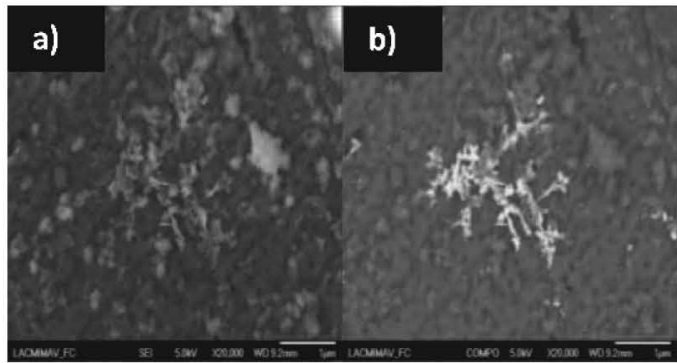

Figura 5. Imagen de SEM con detector de electrones a) secundarios y b) retrodispersados del depósito de $12 \mathrm{~h}$.

Para corroborar lo observado con el detector de ER la figura 6 muestra la imagen de energía dispersiva de rayos $\mathrm{x}(\mathrm{EDX})$ que nos indica que esos aglomerados que se aprecian con más brillo son efectivamente nanopartículas de plata depositadas.

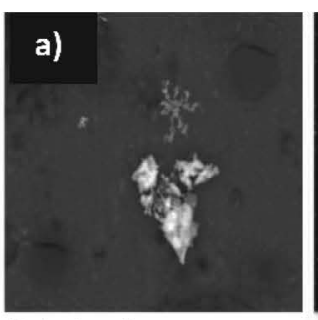

Electron Image 1

Figura 6. Imágenes de a) SEM, b) EDX del depósito de $12 \mathrm{~h}$ de plata.

En la figura 7 se hace la comparación de los tamaños de los depósitos a varios tiempos observando que están aproximadamente entre 7 y $11 \mu \mathrm{m}$, la diferencia entre ellos se puede deber a que no están completamente vertical y lo que da una imagen que aparenta un menor espesor.

Se puede observar que en la imagen c) que resalta un apartado brilloso sobre la superficie de aproximadamente $3 \mu \mathrm{m}$ lo que nos indica que si hubo infiltración, además se puede apreciar en la imagen b) que se observan pequeños aglomerados en la placa de aluminio lo que se puede deber a que la plata se infiltra por las grietas formadas en los depósitos de $\mathrm{TiO}_{2}$.

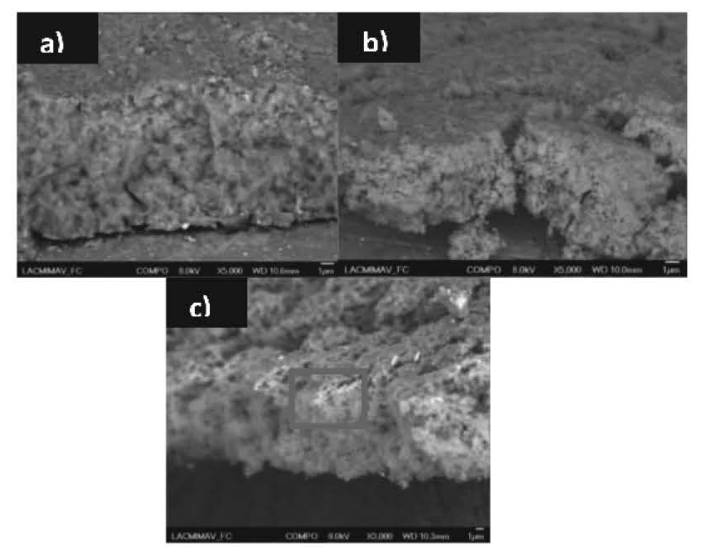

Figura 7. Imagen de SEM de depósitos de $\mathrm{TiO}_{2}$ a a) 0h, b) $6 \mathrm{~h} \mathrm{y}$ c) $12 \mathrm{~h}$ de depósitos de plata.

\section{Conclusiones}

Se obtuvieron depósitos nanoestructurados de $\mathrm{TiO}_{2}$ de $6-11 \mu \mathrm{m}$ de espesor los cuales se observaron mediante SEM. Se sintetizaron nanopartículas de plata con diámetro promedio $25 \mathrm{~nm}$, mediante reducción de iones plata con borohidruro, lo cual se corroboró por medio de espectrofotometría de UV-Vis y energía dispersiva de luz. Las nanopartículas de plata lograron infiltrarse aproximadamente $3 \mu \mathrm{m}$ en el depósito de $\mathrm{TiO}_{2}$, de acuerdo a las imágenes obtenidas por SEM.

\section{Referencias}

1. Corni I, Ryan M P, Boccaccini A R. Electrophoretic deposition: From traditional ceramics to nanotechnology. Journal of the European Ceramic Society 2008; 28 (7): 1353-1367.

2. Boccaccini A R, Roether J A, Thomas B J C, Shaffer M S P, Chavez E, Stoll E, et al. The electrophoretic deposition of inorganic nanoscaled materials. Journal of the Ceramic Society of Japan 2006; 114 (1): 1-14

3. Behnajady M A, Modirshahla N, Shokri M, Rad B. Enhancement of photocatalytic activity of $\mathrm{TiO} 2$ nanoparticles by silver doping: photodeposition versus liquid impregnation methods. Global Nest Journal 2008; 10 (1): 1-7.

4. Huiying Jia, Weiqing Xu, Jing An, Dongmei Li, Bing Zhao A simple method to synthesize triangular silver nanoparticles by light irradiation. Spectrochimica Acta Part A 2006; 64: 956-960. 